\title{
Correlating the Short-Time Current Response of a Hydrogen Evolving Nickel Electrode to Bubble Growth
}

\author{
Nakul Pande, ${ }^{1,2}$ Guido Mul, ${ }^{1}$ Detlef Lohse, ${ }^{2}$ and Bastian Mei $\oplus^{1, *, z}$ \\ ${ }^{I}$ PhotoCatalytic Synthesis Group, Faculty of Science and Technology, MESA ${ }^{+}$Institute for Nanotechnology, University \\ of Twente, 7500 AE Enschede, The Netherlands \\ ${ }^{2}$ Physics of Fluids Group, Faculty of Science and Technology, Max Planck - University of Twente Center for Complex \\ Fluid Dynamics, MESA ${ }^{+}$Institute for Nanotechnology, and J. M. Burgers Centre for Fluid Dynamics, University of \\ Twente, 7500 AE Enschede, The Netherlands
}

\begin{abstract}
Gas evolving electrochemical reactions induce bubble formation and growth at surfaces of electrodes. To study one such situation, hydrogen evolution on nickel electrodes, short time chronoamperometric experiments were performed in combination with in-situ microscopy. The entire electrode of $3.14 \mathrm{~mm}^{2}$ was imaged with confocal microscopy and the current response of the electrode then correlated to the observed bubble growth features. Somehow counterintuitively, first a 2-3\% increase in current was observed consistently when a bubble grows close to the electrode on the edge of the electrode holder, made of a polymer. This is argued to be due to the removal of surface attached gas from the electrode. Next, we observe a consecutive regime of decreasing current, in which large bubbles accumulate on the surface. Interestingly, when these surface attached bubbles coalesce, a steep change in current is observed, which is accompanied by a burst of small bubbles nucleating on the surface previously occupied by the large bubble. These phenomena are qualitatively discussed on the basis of existing literature, and implications for improvements for electrodes on which gases are produced, are outlined.

(C) The Author(s) 2019. Published by ECS. This is an open access article distributed under the terms of the Creative Commons Attribution 4.0 License (CC BY, http://creativecommons.org/licenses/by/4.0/), which permits unrestricted reuse of the work in any medium, provided the original work is properly cited. [DOI: 10.1149/2.0191910jes]

(cc) BY
\end{abstract}

Manuscript submitted November 21, 2018; revised manuscript received January 11, 2019. Published May 30, 2019.

Electrochemical hydrogen production using water electrolysis has been an increasingly important area of study in relation to solutions for storage of renewable energy. Like in any other gas-evolving chemical reaction, during electrochemical hydrogen evolution bubble formation and growth at the electrode surface can occur. ${ }^{1,2}$ Gas molecules produced at the electrode surface initially form discrete domains of surface attached gas, also called 'surface nanobubbles' ${ }^{3-7}$ These nanobubbles grow and coalesce to form micron-size bubbles, 'microbubbles', before finally detaching. ${ }^{4}$

Because of its ubiquity, the growth dynamics of such microbubbles on surfaces has been an active area of research be it in electrolysis, catalysis, or simply by gas oversaturation. ${ }^{8-15}$ In the context of electrochemical systems specifically, bubbles sticking to the electrode surface are generally considered to be detrimental ${ }^{1,2,16-18}$ for the overall cell performance. It therefore is crucial to understand how the gas evolution, the current, and bubble growth are correlated. In particular, it becomes critical to disentangle the interplay between the current at the electrode and the bubble growth.

Given this context, there has been significant research in the past to measure the growth rates of such electrochemically generated gas bubbles. ${ }^{11,12,19}$ The bubble growth rates are commonly parameterized as ${ }^{9,12,19}$

$$
R=\beta t^{x}
$$

where $R$ the radius of the bubble, described as function of time $t$; $x$ is the so-called time coefficient, $\beta$ the so-called growth coefficient. Depending on the ratio of the bubble to the electrode size, broadly, two limiting cases have been identified: diffusion controlled $\left(x=\frac{1}{2}\right)$ and surface reaction controlled ${ }^{9,12}\left(x=\frac{1}{3}\right)$, corresponding to Damköhler numbers $\mathrm{Da}>>1$ and $\mathrm{Da}<<1$, respectively.

At the same time though, some studies have looked at the effect of bubbles on the electrochemical properties of the cell. ${ }^{16,20}$ Sides \& Tobias, ${ }^{21}$ solving for the potential distribution around a bubble, have shown that the primary current around the bubble is depressed. Following up on this work, Dukovic \& Tobias $^{20}$ used numerical simulations to explore the effect of attached bubbles on the current distribution and over-potential. They solved a set of coupled steady-state partial differential equations for the concentration of the dissolved gas and the electrode potential for a base case of hydrogen evolution on a

*Electrochemical Society Member

${ }^{\text {z} E-m a i l: ~ b . t . m e i @ u t w e n t e . n l ~}$ nickel electrode. When treating the combined effects of surface overpotential and dissolved gas concentration, they noted that the current density was highest at the point of attachment of the bubble on the electrode. They explained this counterintuitive enhancement effect as arising from a depression in the dissolved gas concentration, which ultimately suppresses the concentration over-potential at the electrode. Therefore, while it is recognizable that surface coverage due to bubbles may hinder the overall reaction rate, this enhancement effect is clearly counterintuitive. Among more recent works, are experiments that have measured the electrical response at a hydrogen evolving platinum electrode, simultaneously imaging bubbles. ${ }^{13,14}$ While these measurements allowed bubble growth rates to be determined accurately, the side-view images make it difficult to correlate the electrical response to the bubble growth.

To try and address this issue, we used a home-built electrochemical cell allowing for optical characterization of the entire working electrode surface. Unlike the other studies on this subject in the past, ${ }^{9,11,16}$ an inverted working electrode is used in the cell. This cell design inhibited gravity-induced bubble detachment and any resulting rising bubble-induced bulk liquid motion. Furthermore, polydimethylsiloxane (PDMS) was used to confine the working electrode, additionally acting as the weakest spot for bubble nucleation out of hydrogen oversaturated solutions.

With this specific set-up the chronoamperometric response was correlated to bubble growth. While bubbles primarily nucleate near the working electrode at early stages of electrolysis, only at later stages growth of micron-sized bubbles on the working electrode was observed. The inverted electrode design helped to disentangle the current response of the bubble growth from that of bubble detachment induced fluid motion. Moreover, a top-view measurement (in contrast to a side-view projection usually employed in literature) helped to better locate the bubble on the electrode, and correlate it with the current obtained.

Our measurements highlight the role of bubbles near the gasevolving electrode in increasing the current, by possibly removing dissolved gas from the electrode surface. Our measurements, thus, imply that the design of structured electrodes (by placing sites of bubble growth, i.e. hydrophobic nucleation sites, adjacent to catalytically active spots) would increase the cell efficiency, something that has been suggested in the past for porous electrodes, ${ }^{22}$ and employed most recently in the context of bubbles by van der Linde et al. ${ }^{9,23}$ for planar electrodes. 


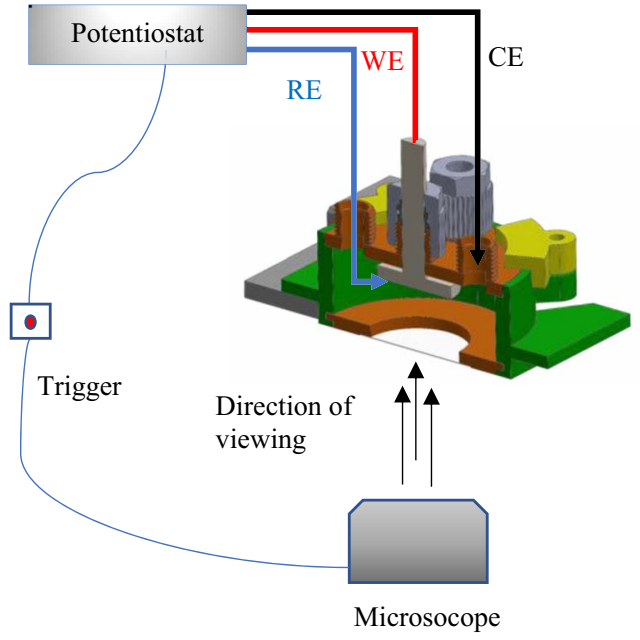

(a)

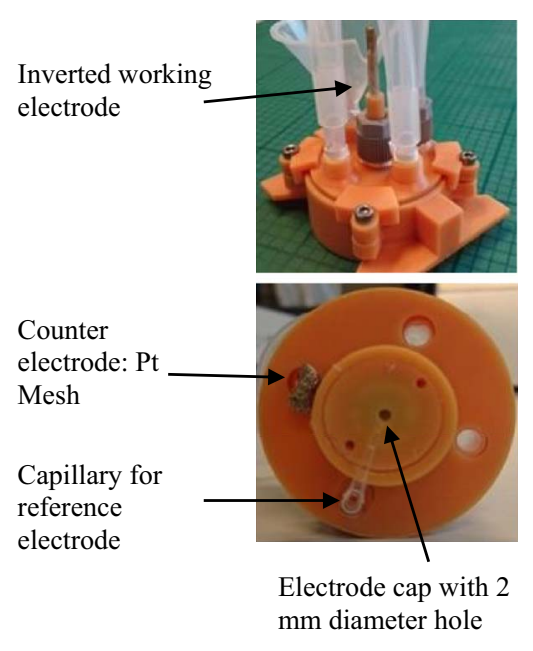

(b)

Figure 1. (a) Shows the schematic of the experimental setup. The microscope images the electrode through a transparent glass window at the bottom of the cell. The working (red), reference (blue) and counter (black) electrodes are connected to the potentiostat which is triggered together with the microscope. (b) shows an image of the assembled cell (top). The inverted working electrode is highlighted. The bottom image shows working, reference and counter electrode assembly. A $2 \mathrm{~mm}$ electrode cap blocks the rest of the electrode from participating in electrolysis and is labelled here (along with the Pt mesh and reference electrode capillary).

\section{Experimental}

Electrochemical cell.—A home-built three-electrode cell (Figure 1) with $0.05 \mathrm{M}$ sodium phosphate buffer solution ( $\mathrm{pH} 6.9$ ) as the electrolyte was used in the experiments. The cell was printed (Rapidshape GP-101 resin) to required design. The working electrode was a nickel disc polished with 0.3 micron alumina paste. The electrochemical cell was fabricated such that only a portion of the working electrode was exposed to the electrolyte. This was done by designing a special cap for the working electrode with a $2 \mathrm{~mm}$ diameter hole (shown in Figure $1 b$ ), and lining the inner surface with polydimethylsiloxane (PDMS). PDMS, being inherently hydrophobic, ensured a good, leakproof fit. The counter electrode used is a platinum mesh, with an area much larger than the working electrode. $\mathrm{Ag} / \mathrm{AgCl}$ electrode (BASi, in $3 \mathrm{M} \mathrm{NaCl}$ ) with an attached glass capillary acted as reference.

Chronoamperometric measurements were performed with a VersaStat 3 potentiostat (Princeton Applied Research) at a fixed potential of $-2 \mathrm{~V}$ with respect to the $\mathrm{Ag} / \mathrm{AgCl}$ reference electrode. The resulting current response was analyzed and correlated to the evolved bubbles. Here the absolute value of the current density has been used in the presented figures. After each measurement, the entire electrode was covered as bubbles did not detach from the electrode surface. The cell was degassed at $0.4 \mathrm{~atm}$, for 30 minutes before and in between successive experiments to remove surface-attached bubbles.

Confocal microscopy.-Bubbles growing on the inverted working electrode were observed using an inverted laser scanning microscope (Nikon confocal microscope A1 system, Nikon Corporation, Tokyo, Japan) with a $4 \mathrm{x}$ dry objective (CFI Plan Apo Lambda 4x/0.2, numerical aperture $=0.2$, working distance $=20 \mathrm{~mm}$ ). The microscope was used in a reflection configuration, ${ }^{24}$ so the metal electrode appeared as a bright spot in the images. A $561 \mathrm{~nm}$ laser was used with the microscope focused on the electrode surface. The images were obtained at about 10 frames/second. They were $512 \times 512$ pixels in size with a scale of $6.23 \mu \mathrm{m} /$ pixel.

\section{Results and Discussion}

Figure 2a shows the stable cyclic voltammogram obtained for the system under study. The onset potential of hydrogen evolution can be seen to be around $-0.8 \mathrm{~V}$ vs $\mathrm{Ag} / \mathrm{AgCl}$. We therefore choose an operating potential much lower than that, namely $-2 \mathrm{~V}$ vs $\mathrm{Ag} / \mathrm{AgCl}$, so that the hydrogen evolution reaction is kinetically fast, which is amenable for bubble growth. It should be noted that the cyclic voltammogram has been taken on a $1 \mathrm{~mm}$ electrode, therefore with a smaller size electrode cap (as opposed to $2 \mathrm{~mm}$ for the rest of the experiments).

The bubbles appear as a changing shadow on the images of the bright Ni electrode. Figure $2 \mathrm{~b}$ shows a typical image obtained along with current-time curves. Since the shadow shows the projected image of the bubble (red circles) on the electrode, it is a measure of the bubble's maximal diameter, and not of its contact diameter. The absolute current density measured results from the reaction on the bubble-free electrode area (total area - bubble contact area), which is not measured here. The different colors show successive runs (blue-red-black), affirming qualitatively repeatable behavior (Figure $2 b$ ).

As highlighted in Figure 3a the current initially shows a steep drop from around 900 to $600 \mu \mathrm{A}$ (corresponding current density: about 29 to $19 \mathrm{~mA} / \mathrm{cm}^{2}$ ), which is the expected electrical response to a steppotential. $^{25}$ The current then achieves a seemingly steady value for about 20 seconds. Although there is constant hydrogen production, no microbubbles are observed on the electrode itself, as can be seen in Figure 3 (a.i). During this time bubbles likely grow out of $\mathrm{H}_{2}$ oversaturated solution at the PDMS lined electrode edge and the electrode cap polymer. Both materials are hydrophobic and more susceptible to bubble growth. Due to the lack of contrast of the bubble against the PDMS background these bubbles cannot be observed by confocal microscopy.

Following this early phase, we then observe a current increase from about 0.47 to $0.95 \mathrm{~mA} / \mathrm{cm}^{2}$ (a $2.5 \%$ increase over the expected steady-state value). This increase occurs at $\sim 20 \mathrm{sec}$, by which time, 0.018 coulombs of charge, or an equivalent 61.2 nano-moles of hydrogen have been produced at the electrode. At such time, bubbles are seen also to grow at the edges of the electrode. This increase is unexpected, but seen in successive experiments as well, in agreement with the inset of Figure 2b. There is some variation in the steady-state and corresponding current increase in succeeding experiments - varying between an increase of $0.25 \mathrm{~mA} / \mathrm{cm}^{2}$ for the blue curve (a $1.2 \%$ increase over the background), to $0.95 \mathrm{~mA} / \mathrm{cm}^{2}$ for the black curve (a 5.2\% increase over the background). The variation in the different experiments can be explained by a partial hydrogen coverage. The electrode is not removed from the electrolyte in between experiments, and adsorbed hydrogen molecules (surface nanobubbles) might reduce the effective reaction area of the electrode also explaining the monotonic decrease in steady state value of the current. Still it is important 


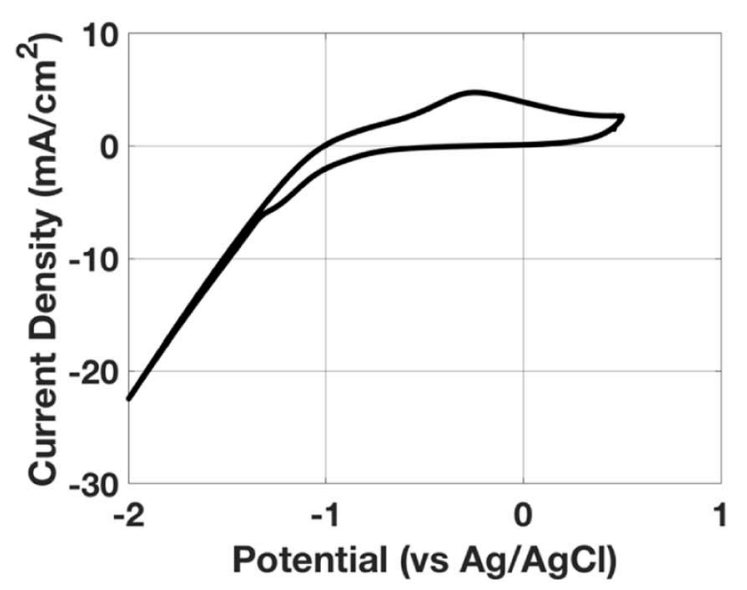

(a)
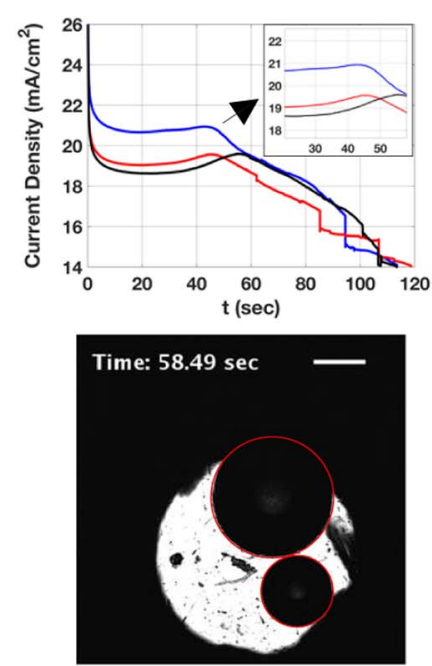

(b)

Figure 2. (a) Cyclic voltammogram of the nickel electrode, of $1 \mathrm{~mm}$ diameter (differently sized electrode cap), in $0.05 \mathrm{M}$ sodium phosphate buffer solution. The operating potential of $-2 \mathrm{~V}$ is clearly in the hydrogen evolution region. (b) Plot of the absolute current density against time for different experiments (different colors). The inset shows a zoomed-in image of the increase. Applied potential: $-2 \mathrm{~V}$ vs Ag/AgCl reference electrode in a $0.05 \mathrm{M}$ sodium phosphate buffer solution (pH 6.9). A typical microscopy image is also shown with a $0.5 \mathrm{~mm}$ scale bar. The bubbles appear as a changing shadow (red circle) on the electrode surface which is the projected image of the bubble on the electrode. It is important to note that only red circled spots are $\mathrm{H}_{2}$ bubbles. All other dark spots visible on the bright electrode are not bubbles but electrode features.

to note that an increase in current density correlates with the growth of a bubble close to the electrode surface on the hydrophobic PDMS.

Figure 3 shows the current density increase, side by side with the images of the electrode at that time, for a typical experiment (Full video in Supplementary information). Natural convection (due to changes in ion concentration close to the electrode) can be excluded due to the design of the experiment. ${ }^{26}$ Thus, following the observations by Dukovic $\&$ Tobias, ${ }^{20}$ this observed increase could be attributed to the removal of dissolved gas close to the electrode surface by this "scavenger" bubble. A schematic of the possible mechanism is shown in Figure 4. In the absence of the bubble, part of the surface is blocked by the formed gas, preventing any reaction at those locations. The presence of the bubble increases the flux of gas away from the electrode, thereby allowing larger availability of reaction sites for $\mathrm{H}_{2} \mathrm{O} / \mathrm{H}^{+}$-ions at the foot of the bubble. This current enhancement thanks to the bubbles will be discussed in more detail in the following section.

Quantitatively, the increase in gas evolution can be compared with the fraction of gas going into the neighboring bubble, i.e. the bubble growing at the perimeter of the electrode. Assuming the bubble to be a perfect sphere (the bubble resembles more of a spherical cap dictated by its contact angle, but this serves as a rough estimate), the rate of gas intake by the bubble is given by its change in mass

$$
\rho \frac{d V}{d t}=\frac{\rho}{M} 4 \pi R^{2} \frac{d R}{d t}\left[\frac{\text { moles }}{s e c}\right],
$$

where $\mathrm{M}$ is the molar mass of hydrogen. Clearly the gas evolution rate is in good agreement with the calculated intake of the idealized bubble as shown in Fig. S1.

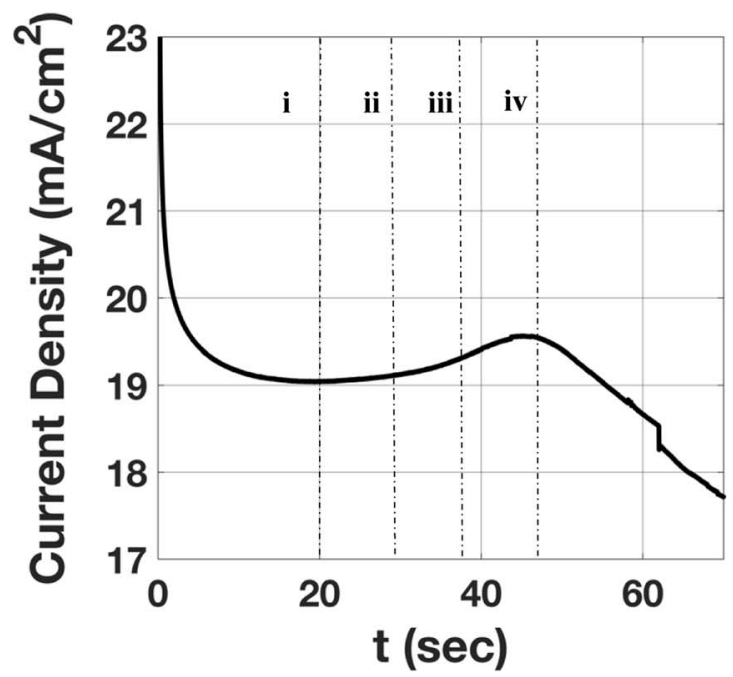

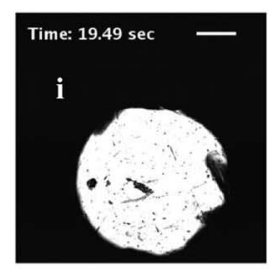
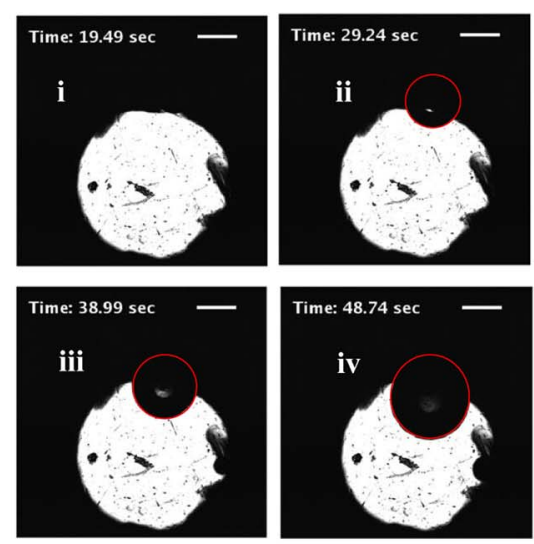

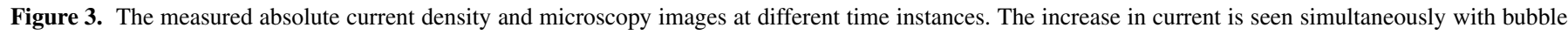
growth from the edge of the electrode. Scale bar: $0.5 \mathrm{~mm}$. 
Gas clusters blocking electrode surface

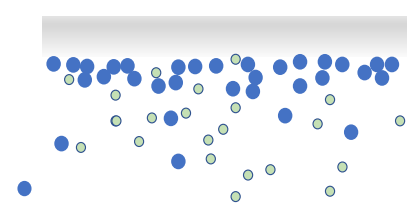

Flux of gas to the bub

freeing up reaction sites

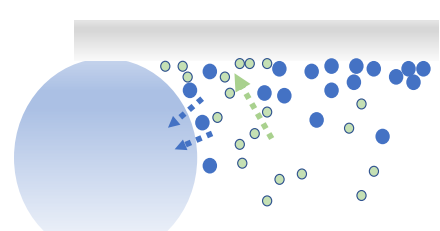

- $\mathrm{H}^{+}$ion /

$\mathrm{H}_{2} \mathrm{O}$ molecule

- $\mathrm{H}_{2}$ molecule

Figure 4. Schematic of the possible mechanism responsible for increased current, highlighted in the box. Left: The case without the bubble, corresponding to 3 (i). Right: The case with the bubble, corresponding to 3 (ii). The bubble removes surface attached gas close to its contact point with the electrode, thereby freeing up reaction sites for hydrogen ions. The arrows indicate the proposed direction of the ion/gas motion.

Further on, the current reaches a peak value and finally decreases. There is likely to be a competition between the current increase due to removal of surface attached gas (or nanobubbles), and current decrease because of physical coverage of electrode by the microbubble, as can be seen in Figure 3iv. This would cause the current to peak before eventually decreasing, as more bubbles accumulate on the electrode, effectively blocking the electrode surface.

Another interesting phenomenon can be easily observed in the second phase of the chronoamperometric measurement. Figure 5a shows the current response to bubble coalescence events for two different experiments. The current profile shows steep changes which we show to correlate (Figure 5) with instances of bubble coalescence. The process of coalescence is sometimes followed by the growth of multiple small bubbles on the electrode. Figure 5 (a.i) highlights one such instance.

As more of the electrode surface is covered by the bubbles, the current eventually does start to decrease. Remarkably, different experiments have a similar slope (Figure 2b). After about 80 seconds, the image of the electrode surface was obscured by the growing bubble, preventing any further observations.

Bubble induced current increase.-Current increase during bubble growth has been observed in recent experiments by other groups as well. ${ }^{13,14}$ In experiments on bubble growth on $\sim 100$ micron platinum electrodes, Fernandez et al. ${ }^{14}$ and Yang et al. ${ }^{13}$ observed a decrease in over-potential and an increase in current respectively while the bubble was growing. Yang et al. ${ }^{13}$ explained this as resulting from the reduction in the bubble covered electrode area. In contrast to this, in our experiments, a bubble is seen growing from the edge, toward the electrode when we see a current increase. Therefore, a decrease in bubble covered electrode area is doubtful. Considering the schematic shown in Figure 4, the removal of surface attached gas could explain the increase in current following the appearance of the bubbles in the vicinity of the electrode. Given the inverted design of the experiment, the physical phenomenon most likely governing this scavenging is diffusion. Close to the electrode, the diffusive flux is $\sim D \frac{C_{\text {electrode }}-c_{s}}{\delta}$. Here $c_{\text {electrode }}$ is the gas concentration at the electrode surface and $c_{s}$ is the saturation gas concentration at bubble surface. Thus, the flux increases drastically for decreasing distance $\delta$ between the electrode and a bubble. It is this flux that removes the surface attached gas, freeing the electrode surface for further electrolysis. This hypothesis is further bolstered by experiments measuring the formation of hydrogen nanobubbles on HOPG (Highly Oriented Pyrolytic Graphite) surfaces, ${ }^{4}$ where, using tapping mode AFM, a reduction in the number of nanobubbles (or surface attached gas clusters) was seen when a microbubble appeared on the electrode surface.

In the electrochemical literature, it is known that supersaturation of the electrolyte with product gas, or the depletion of the reacting ion, locally at the electrode surface, results in an overpotential component related to these concentrations..$^{16,20,27,28}$ Hence, any reduction of gas concentration at the electrode surface (a phenomena that would occur close to the bubble on the electrode) results in a lowering of the concentration overpotential, thereby enhancing the current. ${ }^{20,27}$ In an analogous problem of vapor bubbles produced during boiling, this enhancement effect manifests as cooling of the substrate during bubble growth in boiling, ${ }^{29,30}$ due to the required latent heat.

Current response to bubble coalescence events.-To correlate the current response to bubble coalescence events, we tracked the total projected area of the bubbles from the images obtained for a particular experiment. This could be easily done by tracking the area of the growing shadow on the image. Any sudden changes in this area should indicate bubble coalescence. As anticipated, the abrupt changes in current followed from changes in projected bubble area. This can be seen in Figure 5b.

Yang et al. ${ }^{13}$ saw sharp changes in current when bubbles detached from the electrode surface. Similarly, in our case, after bubble coalescence, the electrode surface is bubble-free. Since there are possibly two competing effects at play here, their joint effect on the current is not straightforward. The electrode surface equivalent to the bubble contact area is freed because of bubble detachment. This should lead to an increase in current at the electrode. On the other hand though, the electrode comes into contact with the supersaturated electrolyte, which leads to nucleation of many small, novel bubbles, which likely inhibit the current. Altogether, the current is susceptible to sharp changes following the removal of a bubble from its surface.

When after coalescence a bubble disappears, the nucleation of multiple small bubbles on the electrode surface is not surprising. The already gas supersaturated electrolyte on coming in contact with the gas-producing electrode surface leads to the sudden eruption of bubbles. This, again, is seen in experiments by Yang et al. ${ }^{13}$ after bubble detachment.

Since the contact area of any of the bubbles could not be measured directly in this experiment, their direct electrode coverage and effect on current changes is an open question and a subject of ongoing research.

\section{Conclusions and Outlook}

In this paper, we have reported the results of short time chronoamperometric experiments on an inverted nickel electrode. The current is intricately linked with the dynamics of bubbles that grow on its surface. Coalescence events have a sharp and abrupt signature on the current measured. Moreover, direct evidence of the increase in current because of a pre-existing "scavenger bubble" has been presented.

We hypothesize that bubbles increase the current by removing dissolved and surface attached gas from the electrode, increasing the number of available reaction sites. This increase in reaction rate close to the bubble, should be reflected as non-uniformity in the ion flux at the electrode as well. Finding the spatial distribution of the ion concentration in the solution near the electrode surface will indeed confirm this hypothesis, and is currently being investigated.

There has been a long-held notion that bubbles only impact the electrolytic cell negatively; ${ }^{1,2}$ but now we have shown an instance where it is otherwise. Our results also suggest a way of designing efficient electrodes: having a patterned surface, with air cavities or 

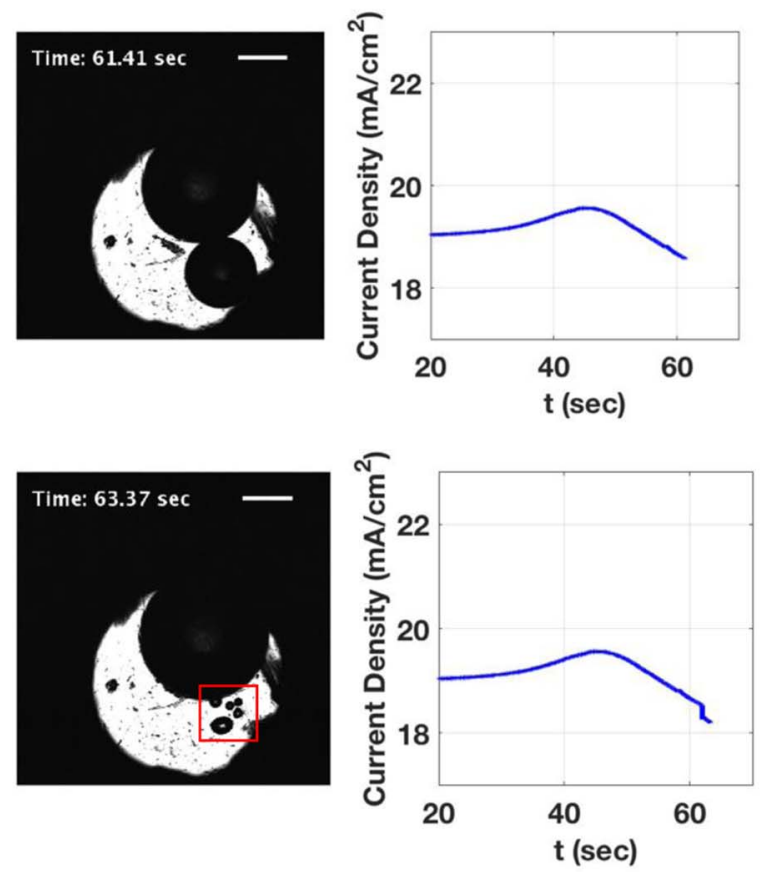

i
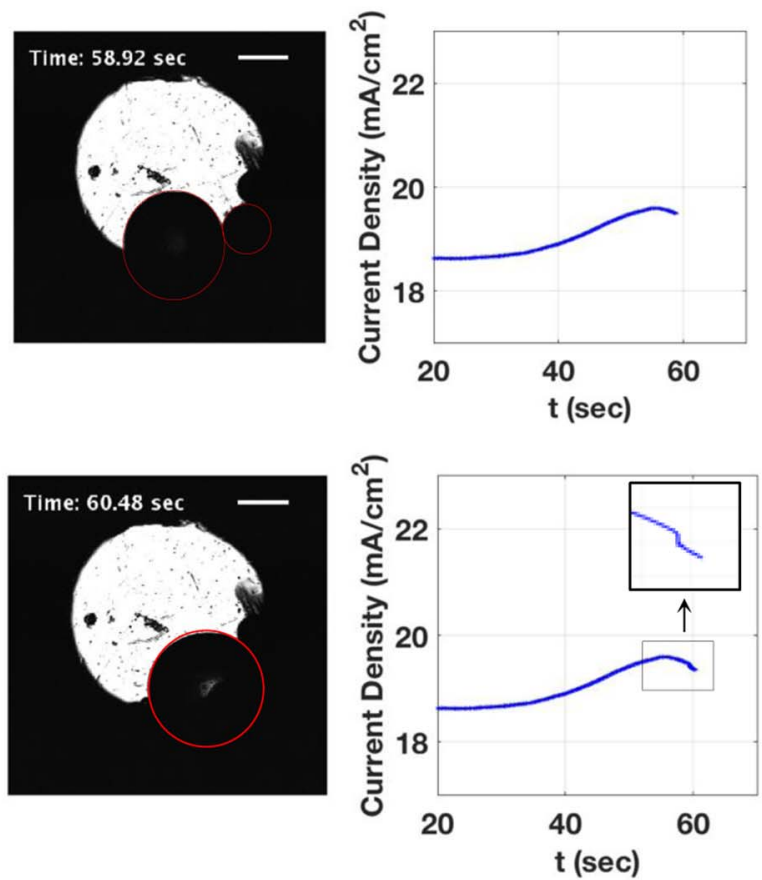

ii

(a)

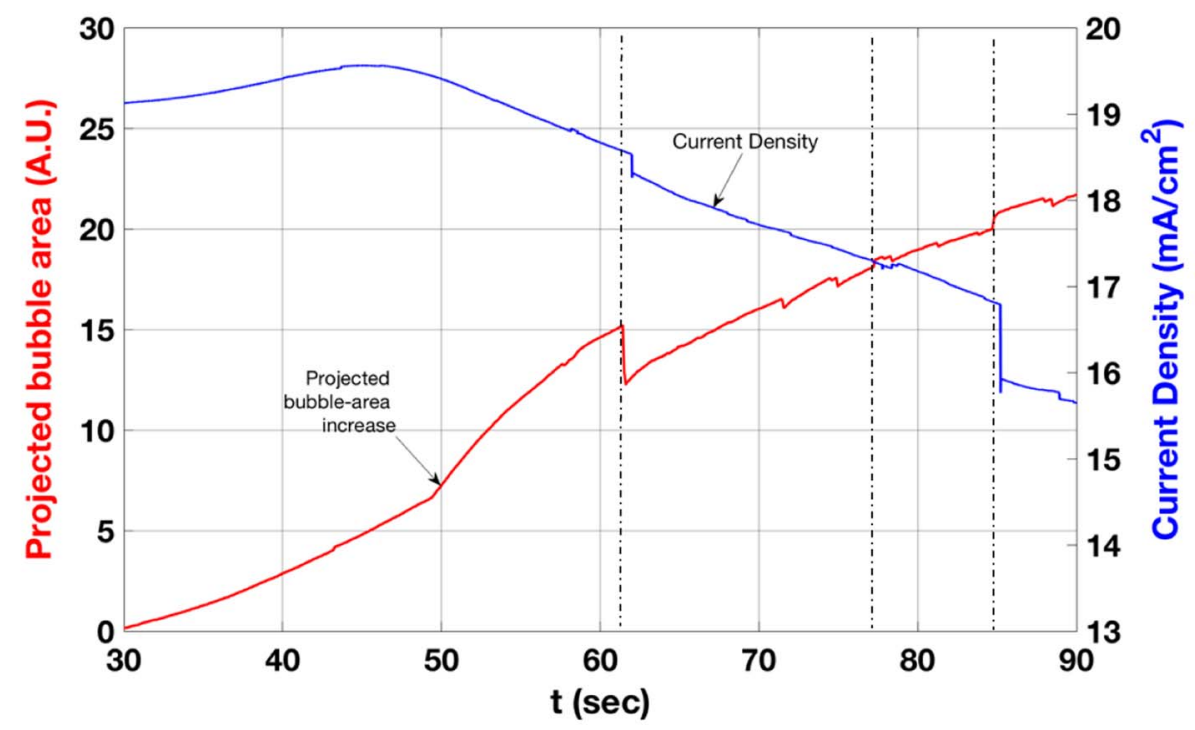

(b)

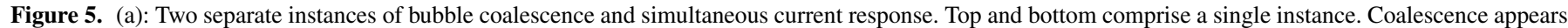

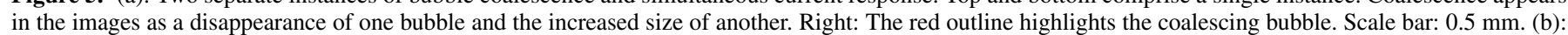

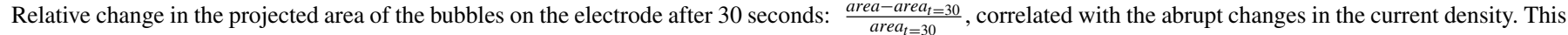

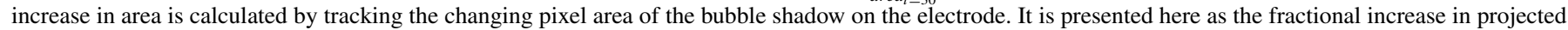
bubble area (arbitrary units A.U.) after $\mathrm{t}=30 \mathrm{sec}$. The black vertical lines highlight the times when abrupt changes in area are followed by change in current.

hydrophobic spots placed adjacent to catalytically active sites. Such a design should allow for greater current with optimal catalyst use.

\section{Acknowledgments}

This research received funding from The Netherlands Organization for Scientific Research (NWO) in the framework of the fund New Chemical Innovations, project ELECTROGAS (731.015.204), with financial support of Akzo Nobel Chemicals, Shell Global Solutions, Magneto Special Anodes (an Evoqua Brand), and Elson Technologies. 


\section{References}

1. K. Zeng and D. Zhang, Recent Progress in Alkaline Water Electrolysis for Hydrogen Production and Applications. Prog. Energy Combust. Sci., 36(3), 307 (2010).

2. M. Wang, Z. Wang, X. Gong, and Z. Guo, The Intensification Technologies to Water Electrolysis for Hydrogen Production - A Review. Renew. Sustain. Energy Rev., 29, 573 (2014).

3. D. Lohse and X. Zhang, Surface Nanobubbles and Nanodroplets. Rev. Mod. Phys., 87(3), 981 (2015).

4. L. Zhang, Y. Zhang, X. Zhang, Z. Li, G. Shen, M. Ye, C. Fan, H. Fang, and J. Hu, Electrochemically Controlled Formation and Growth of Hydrogen Nanobubbles. Langmuir, 22(19), 8109 (2006).

5. S. Yang, P. Tsai, E. S. Kooij, A. Prosperetti, H. J. W. Zandvliet, and D. Lohse, Electrolytically Generated Nanobubbles on Highly Orientated Pyrolytic Graphite Surfaces. Langmuir, 25(3), 1466 (2009).

6. S. Yang, P. Tsai, E. S. Kooij, A. Prosperetti, and J. W. Zandvliet, H.; D. Lohse, Correction to Electrolytically Generated Nanobubbles on Highly Orientated Pyrolytic Graphite Surfaces. Langmuir, 29(19), 5937 (2013).

7. Q. Chen, L. Luo, and H. S. White, Electrochemical Generation of a Hydrogen Bubble at a Recessed Platinum Nanopore Electrode. Langmuir, 31(15), 4573 (2015).

8. O. R. Enríquez, C. Sun, D. Lohse, A. Prosperetti, and D. van der Meer, The QuasiStatic Growth of Bubbles. J. Fluid Mech., 741, R1 (2014).

9. P. van der Linde, Á. Moreno Soto, P. Peñas-López, J. Rodríguez-Rodríguez, D. Lohse, H. Gardeniers, D. van der Meer, and D. Fernández Rivas, Electrolysis-Driven an Pressure-Controlled Diffusive Growth of Successive Bubbles on Microstructured Surfaces. Langmuir, 33(45), 12873 (2017).

10. Á. Moreno Soto, T. Maddalena, A. Fraters, D. van der Meer, and D. Lohse, Coalescence of Diffusively Growing Gas Bubbles. J. Fluid Mech., 846, 143 (2018).

11. J. P. Glas and J. W. Westwater, Measurements of the Growth of Electrolytic Bubbles. ht. J. Heat Mass Transjer, 7, 1427 (1964).

12. N. P. Brandon and G. H. Kelsall, Growth Kinetics of Bubbles Electrogenerated at Microelectrodes. J. Appl. Electrochem., 15(4), 475 (1985).

13. X. Yang, F. Karnbach, M. Uhlemann, S. Odenbach, and K. Eckert, Dynamics of Single Hydrogen Bubbles at a Platinum Microelectrode. Langmuir, 31(29), 8184 (2015).

14. D. Fernández, P. Maurer, M. Martine, J. M. D. Coey, and M. E. Möbius, Bubble Formation at a Gas-Evolving Microelectrode. Langmuir, 30(43), 13065 (2014).

15. P. Lv, H. Le The, J. Eijkel, A. Van den Berg, X. Zhang, and D. Lohse, Growth and Detachment of Oxygen Bubbles Induced by Gold-Catalyzed Decomposition of Hydrogen Peroxide. J. Phys. Chem. C, 121(38), 20769 (2017).
16. C. Gabrielli, F. Huet, M. Keddam, A. Macias, and A. Sahar, Potential Drops Due to an Attached Bubble on a Gas-Evolving Electrode. J. Appl. Electrochem., 19(5), 617 (1989).

17. H. Vogt, The Incremental ohmic Resistance Caused by Bubbles Adhering to an Electrode. J. Appl. Electrochem., 13, 87 (1983).

18. S. Ardo, D. Fernandez Rivas, M. A. Modestino, V. Schulze Greiving, F. F. Abdi, E. Alarcon Llado, V. Artero, K. Ayers, C. Battaglia, J.-P. Becker et al. Pathways to Electrochemical Solar-Hydrogen Technologies. Energy Environ. Sci., 11(10), 2768 (2018).

19. Y. Wang, X. Hu, Z. Cao, and L. Guo, Investigations on Bubble Growth Mechanism during Photoelectrochemical and Electrochemical Conversions. Colloids Surfaces A Physicochem. Eng. Asp., 505, 86 (2016).

20. J. Dukovic and C. W. Tobias, The Influence of Attached Bubbles on Potential Drop and Current Distribution at Gas-Evolving Electrodes. J. Electrochem. Soc., 134(2), 331 (1987).

21. P. J. Sides and C. W. Tobias, Primary Potential and Current Distribution Around a Bubble on an Electrode. J. Electrochem. Soc., 127(2), 288 (1980).

22. T. Kadyk, D. Bruce, and M. Eikerling, How to Enhance Gas Removal from Porous Electrodes? Sci. Rep., 6(1), 38780 (2016).

23. P. van der Linde, P. Peñas-López, Á. Moreno Soto, D. van der Meer, D. Lohse, H. Gardeniers, and D. Fernández Rivas, Gas Bubble Evolution on Microstructured Silicon Substrates. Energy Environ. Sci., (2018).

24. S. Paddock, Confocal Reflection Microscopy: The "Other" Confocal Mode. BioImaging, 32(2), 274 (2002).

25. A. J. Bard and L. R. Faulkner, Electrochemical Methods: Fundamental and Applications, 2nd ed.; 2001.

26. K. Ngamchuea, S. Eloul, K. Tschulik, and R. G. Compton, Advancing from Rules of Thumb: Quantifying the Effects of Small Density Changes in Mass Transport to Electrodes. Understanding Natural Convection. Anal. Chem., 87(14), 7226 (2015).

27. C. Gabrielli, F. Huet, and R. P. Nogueira, Fluctuations of Concentration Overpotential Generated at Gas-Evolving Electrodes. Electrochim. Acta, 50, 3726 (2005).

28. J. A. Leistra and P. J. Sides, Voltage Components at Gas Evolving Electrodes. $J$. Electrochem. Soc., 134(10), 2442 (1987).

29. M. G. Cooper and A. J. P. Lloyd, The Microlayer in Nucleate Pool Boiling. Int. J. Heat Mass Transf., 12(8), 895 (1969).

30. V. K. Dhir, Boiling Heat Transfer. Annu. Rev. Fluid Mech., 30(1), 365 (1998). 